\title{
Quasiparticle Behavior in Tunnel Junction Refrigerators
}

\author{
J.N. Ullom, P.A. Fisher
}

This article was submitted to

Low Temperature Physics Conference 22

Helsinki, Finland, August 5-10, 1999

\section{June 8, 1999}

U.S. Department of Energy

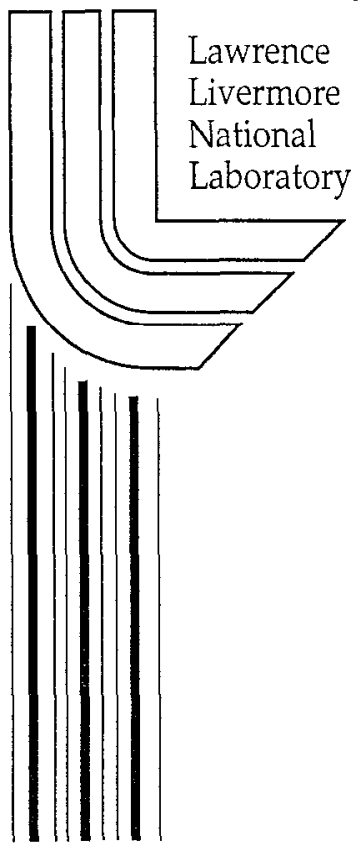




\section{DISCLAIMER}

This document was prepared as an account of work sponsored by an agency of the United States Government. Neither the United States Government nor the University of California nor any of their employees, makes any warranty, express or implied, or assumes any legal liability or responsibility for the accuracy, completeness, or usefulness of any information, apparatus, product, or process disclosed, or represents that its use would not infringe privately owned rights. Reference herein to any specific commercial product, process, or service by trade name, trademark, manufacturer, or otherwise, does not necessarily constitute or imply its endorsement, recommendation, or favoring by the United States Government or the University of California. The views and opinions of authors expressed herein do not necessarily state or reflect those of the United States Government or the University of California, and shall not be used for advertising or product endorsement purposes.

This is a preprint of a paper intended for publication in a journal or proceedings. Since changes may be made before publication, this preprint is made available with the understanding that it will not be cited or reproduced without the permission of the author.

This report has been reproduced

directly from the best available copy.

Available to DOE and DOE contractors from the Office of Scientific and Technical Information

P.O. Box 62, Oak Ridge, TN 37831

Prices available from (423) 576-8401

http://apollo.osti.gov/bridge/

Available to the public from the

National Technical Information Service

U.S. Department of Commerce

5285 Port Royal Rd.,

Springfield, VA 22161

http://www.ntis.gov/

OR

Lawrence Livermore National Laboratory

Technical Information Department's Digital Library

http://www.llnl.gov/tid/Library.html 


\title{
Version Date
}

$6 / 8 / 99$

\section{Title}

quasiparticle behavior in tunnel junction refrigerators

\section{Authors}

J.N. Ullom ${ }^{a}$, P.A. Fisher ${ }^{b}$

\section{Affiliations}

"Lawrence Livermore Laboratory, L418, Livermore CA, 94550, USA

${ }^{\mathrm{b}}$ Analog Devices Corp., MS426, Wilmington MA, 01887, USA

\begin{abstract}
We present simulations of quasiparticle creation, propagation, and loss in the superconducting electrode of a normal-insulator-superconductor tunnel junction refrigerator. We calculate the electronic temperature in the superconducting electrode from self-consistent solutions to the diffusion equation. We then calculate the power load on the normal electrode due to recombination phonons and the reduction in cooling power due to the elevated temperature of the superconductor. Our calculations explain the degraded cooling performance observed in 15-20 $\mu \mathrm{m}$ sized $\mathrm{Ag} / \mathrm{AlOx} / \mathrm{Al}$ junctions.
\end{abstract}

\section{Keywords}

microrefrigeration, quasiparticles, tunnel junctions

\section{Contact Information of Corresponding Author}

J. N. Ullom

Lawrence Livermore National Laboratory

7000 East Ave, L418

Livermore, CA 94550

USA

Fax: 925-424-5512

Email: ullom1@1lnl.gov 
Since the first demonstration of electron cooling by normal-insulatorsuperconductor (NIS) tunnel junctions [1], there has been growing interest in the development of on-chip refrigerators having base temperatures near $100 \mathrm{mK}$ when operated from bath temperatures of several hundred $\mathrm{mK}$. [2,3] In this paper, we show how heating of the electron system in the superconducting electrode of a NIS junction can degrade refrigerator performance.

Current flow through a NIS tunnel junction heats the electron system in the superconducting electrode through the creation of quasiparticles. This heating degrades the cooling performance of the junction through two mechanisms. First, the annihilation of quasiparticles produces phonons which can travel through the tunnel barrier and excite electrons in the normal electrode. Second, the power removed from the normal electrode decreases as the quasiparticle density in the superconductor increases. Electrons which tunnel from below the Fermi level of the normal metal and annihilate hole-like excitations in the superconductor are especially detrimental to the cooling power of the junction. The two degradation mechanisms are described quantitatively below.

If the quasiparticle density in the superconducting electrode of the junction is $n_{x}+n_{t h}$ where $n_{t h}$ is the thermal density, then the recombination rate is $\Gamma_{R}\left(n_{x}+n_{t h}\right)^{2} V_{S}$ quasiparticles per second where $\Gamma_{R}$ is the effective recombination rate per unit density and $V_{S}$ is the electrode volume. The power load on the normal clcctrode duc to recombination phonons is then given approximately by

$$
\Gamma_{R}\left(n_{x}^{2}+2 n_{x} n_{t h}\right) V_{S} \Delta p_{p-e}
$$

where $\Delta$ is the energy gap and $p_{p-e}$ is the probability that a recombination phonon excites an electron in the normal electrode.

The power deposited in the superconducting electrode of a NIS junction is given by 


$$
\begin{array}{r}
P_{S}=\frac{1}{e^{2} R_{N}} \int_{\Delta}^{\infty} E N(E)\left[f_{N}\left(E+e V_{b}\right)+f_{N}\left(E-e V_{b}\right)\right. \\
\left.-2 f_{S}(E)\right] d E
\end{array}
$$

where $R_{N}$ is the normal state resistance, $N(E)$ is the energy-dependence of the density of states, $V_{b}$ is the bias voltage, and $f_{N}\left(f_{S}\right)$ is the Fermi function in the normal (superconducting) electrode. We assume that the electron system in the normal metal (superconductor) has an effective temperature $T_{N}\left(T_{S}\right)$. The cooling power in the normal electrode, $P_{N}$, is $P_{S}-I V_{b}$. In Fig. 1, we plot the normalized cooling power as a function of $T_{S} / T_{N}$ for $T_{N}$ equal to $0.1,0.2$, and $0.3 \mathrm{~K}$ and $\Delta=180 \mu \mathrm{eV}$. It can be seen that the cooling power decreases when $T_{S}$ exceeds $T_{N}$. The loss in cooling power due to heating in the superconductor is given by

$$
P_{N}\left(T_{N}, T_{S}\right)-P_{N}\left(T_{N}, T_{S}=T_{b}\right)
$$

where $T_{b}$ is the bath temperature.

Previous experimental work on 15-20 $\mu \mathrm{m}$ long junctions described deviations from the expected thermal behavior in terms of a power load on the normal electrode $\beta P_{S}\left(T_{N}, T_{S}=T_{b}\right)$. [3] This description implies that a fraction $\beta$ of the power deposited in the superconductor, $P_{S}$, returns to the normal electrode. The fraction $\beta$ was measured to be $0.125-0.15$ for $T_{b}$ between 0.2 and $0.3 \mathrm{~K}$ and $V_{b}$ between 0 and $1.1 \Delta / e$. The same $\beta$ was found for 2 junctions with lengths of 20 and $15 \mu \mathrm{m}$, areas of 400 and $150 \mu \mathrm{m}^{2}$, and specific resistances of $3300 \Omega \mu \mathrm{m}^{2}$ and $7200 \Omega \mu \mathrm{m}^{2}$, respectively. We next describe how to calculate $\beta$.

To estimate the power load due to quasiparticle recombination and the loss in cooling power due to values of $T_{S}>T_{b}$, we calculate the quasiparticle density in the superconducting electrode. This is equivalent to calculating $T_{S}$. The spatially varying excess quasiparticle density, $n_{x}(x)$, is given by 


$$
D \nabla^{2} n_{x}-\Gamma_{R}\left(n_{x}{ }^{2}+2 n_{x} n_{t h}\right)+\Gamma_{Q P} g(x)=0
$$

where $D$ is the diffusion constant. The end of the junction is a mirror and the point where the superconducting lead overlaps a normal contact pad is a sink. The function $g(x)$ is zero except under the junction where $g(x)^{-1}$ is the volume of the superconducting electrode. The rate of quasiparticle injection, $\Gamma_{Q P}$, is given by Eq. (2) without the factor of $E$ inside the integral. Since $\Gamma_{Q P}$ is an integral function of $T_{S}$ and thus $n_{x}$, we solve Eq. (4) iteratively until a self-consistent solution for $n_{x}$ is found.

Calculated values of $n_{x}$ due to currents of $1.67 \mu \mathrm{A}$ (solid line) and $6.84 \mu \mathrm{A}$ (dashed line) are plotted in Fig. 2. These currents correspond to $V_{b}=0.75 \Delta / e$ and $1.0 \Delta / e$, and $T_{N}=0.3022$ and $0.3086 \mathrm{~K}$, respectively. The bath temperature was $0.304 \mathrm{~K}$. The injection region extends from $x=0$ to $x=20 \mu \mathrm{m}$ and the quasiparticle sink occurs at $x=47.5 \mu \mathrm{m}$. The cross-sectional area is $3.4 \mu \mathrm{m}^{2}$. Including phonon trapping, we calculate $\Gamma_{R}=30 \mu \mathrm{m}^{3} / \mathrm{s}$. The diffusion constant $D$ is calculated from resistivity measurements and the quasiparticle dispersion relation. A value for $D$ of $9 \times 10^{9} \mu \mathrm{m}^{2} / \mathrm{s}$ is typical, but there is a weak dependence on $V_{b}, T_{N}$, and $T_{S}$. To simplify, we set $T_{S}$ equal to $T_{b}$ when calculating $D$. The excess densities of $10,500 \mu \mathrm{m}^{-3}$ and $37,300 \mu \mathrm{m}^{-3}$ in the junction region correspond to $T_{S}=0.371$ and $0.447 \mathrm{~K}$, respectively.

Given $n_{x}$ and $T_{S}$ in the superconducting electrode of the junction, it is simple to calculate $\beta$. Using the phonon-electron interaction rate for $2 \Delta$ phonons in $\mathrm{Ag}$ and the phonon escape rate from the $\mathrm{Ag}$ normal electrode, we estimate that $p_{p-e}$ in Eq. (1) is approximately 0.4 . We conclude from Eq. (1) that for $I=1.67 \mu \mathrm{A}$, recombination produces a power load of $4 \mathrm{pW}$ on the normal electrode. The decrease in cooling power due to elevation of $T_{S}$ is also significant. From Eq. (3), we conclude that the elevation of $T_{S}$ above $T_{b}$ reduces the cooling power by $11 \mathrm{pW}$. Summing these powers and dividing 
by Eq. (2) evaluated with $T_{S}=T_{b}$, we arrive at $\beta=.05$. For $I=6.84 \mu \mathrm{A}$, the recombination load is $37 \mathrm{pW}$, the decrease in cooling power is $42 \mathrm{pW}$, and $\beta$ is .06. These predictions for $\beta$ incorporate no free parameters and agree with measurement to factors of 2-3. The predictions are largely independent of bias, in good agreement with measurement. Similar calculations carried out at $T_{b}=0.226 \mathrm{~K}$ yield $\beta=.05$ for $V_{b}=0.75 \Delta / e$ and $\beta=.06$ for $V_{b}=0.9 \Delta / e$. Hence, the predicted values of $\beta$ are independent of $T_{b}$ over the range $0.2-0.3 \mathrm{~K}$, also in good agreement with measurement. The predicted values of $\beta$ can be increased to $0.125-0.15$ by decreasing the mean free path by a factor near 3 .

The predicted values of $\beta$ for the $15 \mu \mathrm{m}$ long junction in [3] are smaller: .02-.03. It is surprising that $\beta$ measured in the $15 \mu \mathrm{m}$ and $20 \mu \mathrm{m}$ junctions is the same because of the higher specific resistance and shorter distance quasiparticles need diffuse before leaving the smaller junction. It is possible that the mean free path in the $\mathrm{Al}$ of the smaller junction is shorter than in the larger because shadow mask deposition produces more disorder in small features.

In conclusion, we have demonstrated techniques for calculating the degradation in NIS refrigerator performance caused by heating of the superconducting electrode. Our calculations are in close quantitative agreement with measurements and show the same insensitivity to bath temperature and bias voltage.

This work was performed under the auspices of the U.S. Department of Energy by Lawrence Livermore National Laboratory under contract no. W-7405-ENG-48.

[1] M. Nahum, T. Eiles, and J. M. Martinis, Appl. Phys. Lett. 65 (1994) 3123.

[2] M.M. Leivo, J.P. Pekola, and D.V. Averin, Appl. Phys. Lett. 68 (1996) 1996, and subsequent papers by the same authors.

[3] P.A. Fisher, J.N. Ullom, and M. Nahum, Appl. Phys. Lett. 74 (1999) 2705. 
Fig. 1. Cooling power $P_{N}$ with normal electrode temperature $T_{N}$ and superconducting electrode temperature $T_{S}$ plotted as a function of $T_{S} / T_{N}$ for $T_{N}=0.1,0.2$, and $0.3 \mathrm{~K}$. The result is normalized by $P_{N}$ evaluated with $T_{S}=T_{N}$. The bias is $0.95 \Delta / e$.

Fig. 2. Excess quasiparticle density $n_{x}(x)$ plotted versus $x$ for junction currents of 1.67 $\mu \mathrm{A}$ and $6.84 \mu \mathrm{A}$. 
Fig. 1.

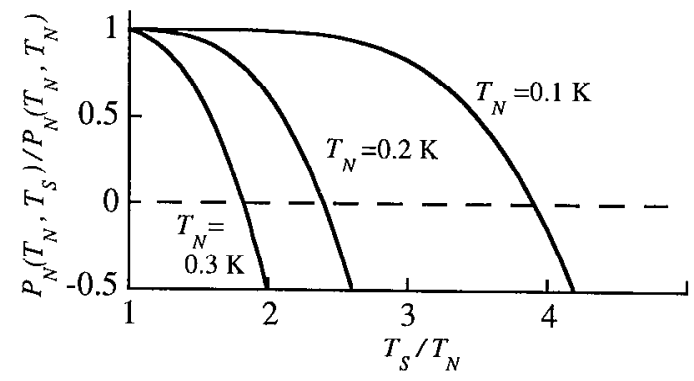


Fig. 2.

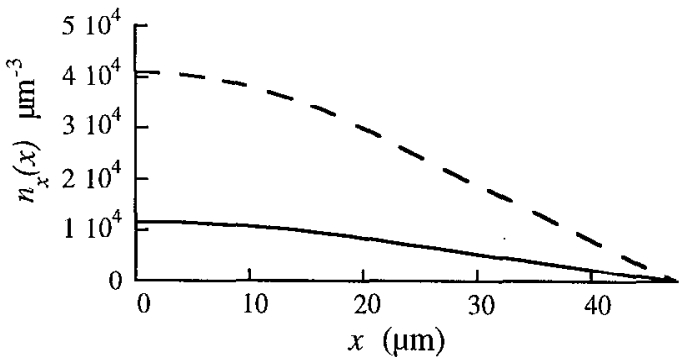

